\title{
Estimation of the Passive Earth Pressure with Inclined Cohesive Backfills: the Effect of Intermediate Principal Stress is Considered
}

 \\ ${ }^{I}$ Key Laboratory of Engineering Geomechanics, Institute of Geology and Geophysics, Chinese Academy of Sciences, \\ Beijing 100029, China \\ ${ }^{2}$ Exploration and Development Research Institute, Daqing Oil Field Company Ltd., Daqing, Heilongjiang 163712, \\ China \\ ${ }^{3}$ Beijing Orangelamp Navigation Technology Development Co., Ltd, Beijing 10085, China \\ ${ }^{4}$ Henan Provincial Communications Planning Survey and Design Institute Co., Ltd. Zhenzhou 450052, China
}

\begin{abstract}
Estimating passive earth pressure accurately is very important when designing retaining wall. Based on the unified strength theory and plane strain assumption, an analytical solution has been developed to determine the passive lateral earth pressure distribution on a retaining structure when the backfill is cohesive and inclined considering the effect of the intermediate principal stress. The solution derived encompasses both Bell's equation (for cohesive or cohesionless backfill with a horizontal ground surface) and Rankine's solution (for cohesionless backfill with an inclined ground surface).
\end{abstract}

Keywords: Passive earth pressure, inclined backfill, unified strength theory, intermediate principal stress.

\section{INTRODUCTION}

Passive earth pressure plays an important role in soilstructure interaction in many structures in civil engineering such as retaining walls, retaining piles around a foundation ditch, and so on. Therefore, estimating the pressure accurately is very useful in geotechnical engineering, especially in the design of simpler retaining structures such as small gravity retaining walls. A theoretical framework for earth pressure theory has been firmly established over the past couple of decades. Classical Rankine passive pressure theory is one of the most important earth pressure theories because of its rigorous theory, clear concept and simple calculation. Therefore, Rankin's theory is still used.

Passive earth pressure, $\sigma_{\mathrm{p}}$, acting on a retaining structure with inclined cohesionless backfill with an angle $\beta$ with the horizontal (with cohesion, $c=0$, and friction angle, $\phi>0$ ) is expressed by Rankine:

$\sigma_{\mathrm{p}}=\mathrm{K}_{\mathrm{p}} \sigma_{\mathrm{z}}$

Here, $\sigma_{z}$ is the vertical effective stress, and $K_{p}$ is the passive earth pressure coefficient, where

$$
K_{\mathrm{p}}=\frac{\cos \beta+\sqrt{\cos ^{2} \beta-\cos ^{2} \phi}}{\cos \beta-\sqrt{\cos ^{2} \beta-\cos ^{2} \phi}}
$$

\footnotetext{
*Address correspondence to this author at the Key Laboratory of Engineering Geomechanics, Institute of Geology and Geophysics, Chinese Academy of Sciences, Beijing 100029, China; Tel: +86-010-82998610 (Office), +86-13426310546 (Mobile); Fax: +86-010-82998610; E-mail: yuwenlong198676@163.com
}

When the backfill is cohesive $(\phi>0, c>0)$ and is horizontal, the passive earth pressure, $\sigma_{\mathrm{p}}$, is calculated using Bell's eq. (3):

$$
\sigma_{\mathrm{p}}=\sigma_{\mathrm{z}} K_{p}+2 c \sqrt{K_{p}}
$$

Where

$$
K_{p}=\tan ^{2}\left(45^{\circ}+\phi / 2\right)
$$

Equations (1)-(4) are valid for smooth vertical walls. Although the back of every real retaining wall is rough, approximate values of the earth pressure can be obtained on the assumption that it is smooth [1]. Therefore, all the retaining walls in this paper are assumed to be smooth and vertical.

There are other theoretical methods to determine the lateral earth pressures when the wall is vertical, in addition to the method of Rankine. Based on the assumption of a logarithmic spiral failure surface, Caquot and Kerisel [2] developed tables of earth pressure coefficients. Sokolovski [3] presented a method based on finite-difference solution. Habibagahi and Ghahramani [4] developed a solution for lateral earth pressure coefficients based on zero extension line theory.

All the above methods can not be used in the case where the soil behind the wall is sloping and consists of soils with cohesion. With the trial wedge (graphical) method NAVFAC [5] determined the active lateral force for each case using a force polygon.

In general, the most favorable backfill materials are permeable coarse-grained soils with well settlement, preferably with little silt or no clay content, but such materials may 
be unavailable or too expensive. So in some areas where well-drained granular soils are in short supply locally available cohesive or poor quality granular soils are used as backfill [1].

Based on multi-slip mechanism and the model of multishear element, M.H.Yu established the unified strength theory (UST) which takes into consideration the different contribution of all stress components to the yield of failure of materials [6, 7]. The UST encompasses the twin shear strength theory $[8,9]$ and single strength theory. The theory agrees with experimental results well over a wide range of stress states for many materials including metal, rock, soil, concrete, and others. So it has been applied successfully in some cases [10-12].

However, little research on passive earth pressure has been done with respect to the intermediate principal stress. J. Zhang [13] has done some work. Further studies are still necessary to calculate passive earth pressure considering all principal stresses.

The purpose of this paper is to determine the passive lateral earth pressure distribution on a retaining structure against an inclined cohesive backfill when considering the intermediate principal stress.

\section{REVIEW OF THE UNIFIED STRENGTH THEORY}

The UST has a unified model and simple unified mathematical expression that is suitable for various materials. The mathematical expression can be introduced as follows $[6,7]$ :

If $\sigma_{2} \leq \frac{\sigma_{1}+\lambda \sigma_{3}}{1+\lambda}$

Then $\sigma_{1}-\frac{\lambda\left(b \sigma_{2}+\sigma_{3}\right)}{1+b}=\sigma_{t}$

Else $\frac{1}{1+b}\left(\sigma_{1}+b \sigma_{2}\right)-\lambda \sigma_{3}=\sigma_{t}$

Where $b$ is coefficient of intermediate principal stress, the parameter, $\lambda$, is the ratio of tensile strength, $\sigma_{t}$, and compressive strength, $\sigma_{\mathrm{c}}$. In geotechnical, $\lambda$ and $\sigma_{\mathrm{t}}$ can be expressed by shear strength parameters:

$\lambda=\frac{\sigma_{t}}{\sigma_{c}}=\frac{1-\sin \phi}{1+\sin \phi}$

$\sigma_{t}=\frac{2 c \cos \phi}{1+\sin \phi}$

According to unified strength theory, the parameter $b$ plays a significant role. With different values of $b$, the unified strength theory represents or approximates all the traditionally strength or yield criteria. Hence, the unified strength theory may be regarded as a theoretical system to cover a series of regular strength criteria. When $b=0 ; 0.5$ and 1 , the Tresca yield criterion, linear approximation of the von Mises yield criterion and the twin-shear stress yield criterion are obtained, respectively [6]. Fig. (1) shows the loci of UST in the deviatoric plane.
Twin-shear theory $(b=1$, Yu1985) Single-shear theory (b=0, Mohr-Coulomb1900)

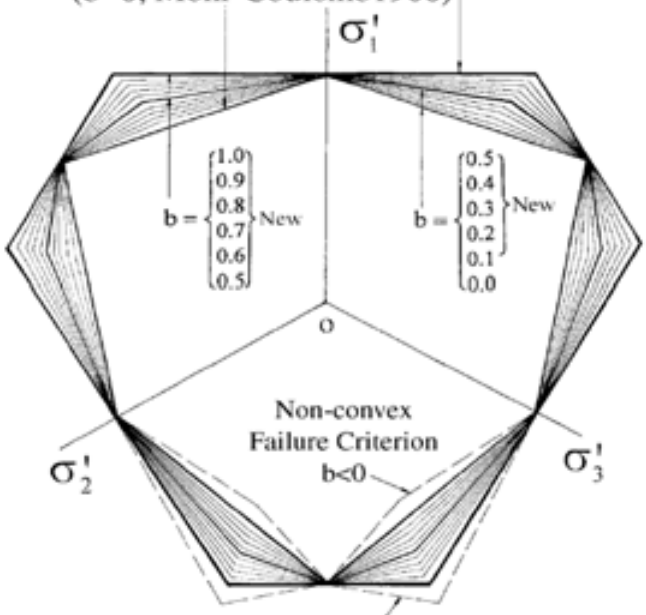

Non-convex Failure Criterion $b>1$

Fig. (1). The loci of the unified strength theory in the deviatoric plane (by M.H. Yu 2001).

Substituting eqs. (7) and (8) into eq.(5) and eq. (6), we get:

If $\sigma_{2} \leq \frac{\sigma_{1}+\sigma_{3}}{2}-\frac{\sigma_{1}-\sigma_{3}}{2} \sin \phi$,

Then,

$\sigma_{3}=\tan ^{2}\left(45^{\circ}-\frac{\phi}{2}\right)(1+b) \sigma_{1}-b \sigma_{2}-2(1+b) \tan \left(45^{\circ}-\frac{\phi}{2}\right) c$

Else,

$\sigma_{3}=\tan ^{2}\left(45^{\circ}-\frac{\phi}{2}\right) \frac{b \sigma_{2}+\sigma_{1}}{1+b}-2 \tan \left(45^{\circ}-\frac{\phi}{2}\right) c$

\section{THEORETICAL ANALYSIS MODEL}

The following conditions are assumed in deriving the analytical solution: (1) the soil is isotropic and homogeneous and has both internal angle of friction and cohesion; the friction and cohesion are constant and remain independent of depth; (2) the force on the wall is acting parallel to the slope.

Consider a retaining wall with a cohesive backfill of slope angle $\beta$ ( $\beta$ is positive when the surface of the backfill slopes upwards from the top of the wall), as shown in Fig. (2). If the backfill of the retaining wall moves to the wall, the force on the wall decreases gradually. On the contrary, if the retaining wall moves to the backfill behind the wall, the force on the wall increases gradually. When the soil behind the wall is in the passive stress state, the pressure acting on the wall is the passive earth pressure. For an element of soil at the dept $z$ at the back of the wall, the construction of Mohr's circle of stresses in passive stress state is shown in Fig. (3). The vertical stress on the soil element is denoted as OA and is expressed as

$$
\mathrm{OA}=\gamma z \cos \beta
$$

Where $\gamma$ is the unit weight of the soil, and $z$ is the depth below ground surface. The lateral stress on the soil element 
is denoted by OB' in Fig. (3). OA and OB' will hereafter be referred to as $\sigma_{z}$ and $\sigma$, respectively. However, it should be noted that in this paper $\sigma_{z}$ and $\sigma$ are not stresses acting normal to their respective planes.

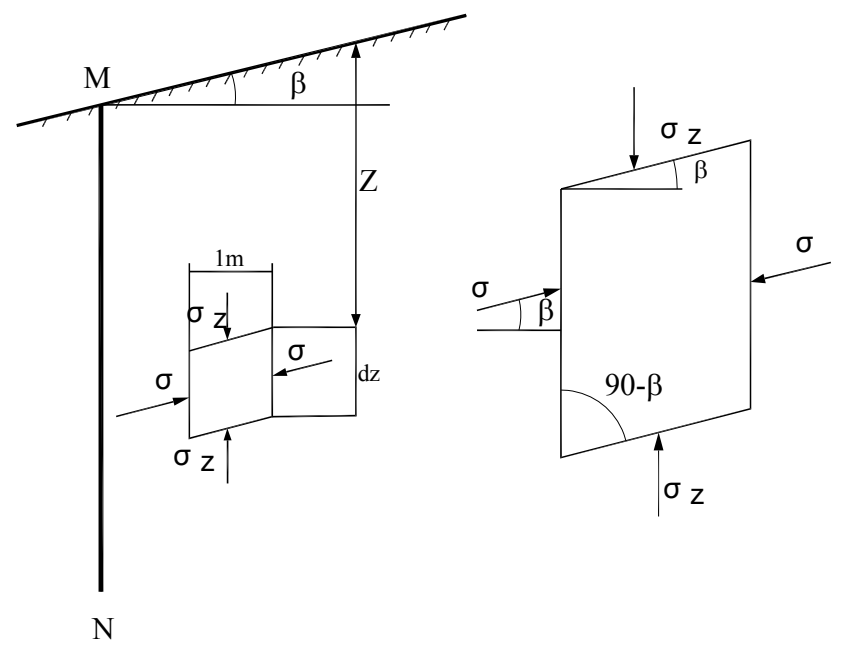

Fig. (2). General soil-structure system.

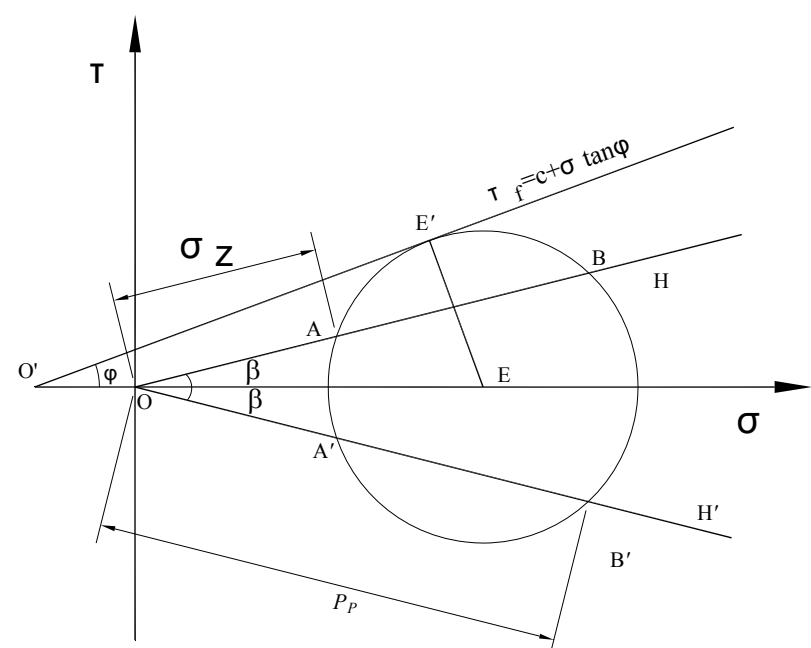

Fig. (3). Mohr's circle to derive the analytical active earth pressure expression.

The equations of the Mohr's circle and Line $\mathrm{OH}$ can be written as, respectively

$\left(\sigma-\frac{\sigma_{1}+\sigma_{3}}{2}\right)^{2}+(\tau-0)^{2}=\left(\frac{\sigma_{1}-\sigma_{3}}{2}\right)^{2}$

$\tau=\sigma \tan \beta$

So we get a unary quadratic equation of $\sigma$ :

$\left(1+\tan ^{2} \beta\right) \sigma-\left(\sigma_{1}+\sigma_{3}\right) \sigma+\sigma_{1} \sigma_{3}=0$

\section{Passive earth Pressure Strength $\mathbf{p}_{p}$}

When the soil is in passive limit equilibrium state, the force is denoted as the passive earth pressure $p_{p}$. As seen from Fig. (3), lines $\mathrm{OH}$ and $\mathrm{OH}^{\prime}$ are symmetrical, the lengths of lines $\mathrm{OA}$ and $\mathrm{OA}^{\prime}$ are the same; the lengths of lines $\mathrm{OB}$ and line OB' are also the same. When the force on the wall is the passive earth pressure, then

$$
\begin{aligned}
P p=\sigma=O B^{\prime}=O B, \sigma_{z}=O A^{\prime} & =O A, \sigma_{A}=\sigma_{z} \cos \beta \\
& =\gamma z \cos ^{2} \beta, \sigma_{B}=p_{p} \cos \beta
\end{aligned}
$$

Where $\sigma_{\mathrm{A}}$ and $\sigma_{\mathrm{B}}$ are the two roots of eq. (13):

$$
\begin{gathered}
\sigma_{A}=\frac{\left(\sigma_{1}+\sigma_{3}\right)-\sqrt{\left(\sigma_{1}+\sigma_{3}\right)^{2}-4 \sigma_{1} \sigma_{3}\left(1+\tan ^{2} \beta\right)}}{2\left(1+\tan ^{2} \beta\right)} \\
\sigma_{\mathrm{B}}=\frac{\left(\sigma_{1}+\sigma_{3}\right)+\sqrt{\left(\sigma_{1}+\sigma_{3}\right)^{2}-4 \sigma_{1} \sigma_{3}\left(1+\tan ^{2} \beta\right)}}{2\left(1+\tan ^{2} \beta\right)}
\end{gathered}
$$

From eqs. (13), (14) and (16), the following equations are obtained:

$$
\begin{aligned}
& \sqrt{\left(\sigma_{1}+\sigma_{3}\right)^{2}-4 \sigma_{1} \sigma_{3}\left(1+\tan ^{2} \beta\right)}=2 \gamma z-\left(\sigma_{1}+\sigma_{3}\right) \\
& \sigma_{1} \sigma_{3}=\left[\gamma z\left(\sigma_{1}+\sigma_{3}\right)-\gamma^{2} z^{2}\right] \cos ^{2} \beta
\end{aligned}
$$

According to eqs. (14), (15) and (16), eq. (19) is obtained:

$$
\sigma_{A}+\sigma_{B}=\frac{\sigma_{1}+\sigma_{3}}{1+\tan ^{2} \beta}=\mathrm{p}_{\mathrm{p}} \cos \beta+\sigma_{\mathrm{z}} \cos \beta
$$

Equating eq. (19) with eq. (17), we get:

$$
\begin{aligned}
p_{\mathrm{p}}=\frac{2\left(\sigma_{1}+\sigma_{3}\right)-2 \gamma z}{2 \gamma z} \sigma_{z} & =\frac{\sigma_{1}+\sigma_{3}}{\gamma z} \sigma_{z}-\sigma_{z} \\
& =\left(\sigma_{1}+\sigma_{3}\right) \cos \beta-\gamma z \cos \beta
\end{aligned}
$$

\section{Definition of Intermediate Principal Stress}

Plane strain state is widely existent in geotechnical engineering such as slope, strip foundation, retaining wall etc. Intermediate principal stress can be determined by using the generalized Hook's law when analyzing the strength and deformation condition of rock and soil body with the nonlinear method. Suppose that the cross section of retaining wall as $\mathrm{x}-\mathrm{z}$ plane, thus the direction vertical to the cross section is $y$-direction. With the elastic solution of plane strain problem,

$$
\varepsilon_{y}=0
$$

According to the generalized Hook's law:

$$
\varepsilon_{y}=\frac{1}{E}\left[\sigma_{y}-v\left(\sigma_{z}+\sigma_{x}\right)\right]
$$

In $x-z$ plane: $\sigma_{z}+\sigma_{x}=\sigma_{1}+\sigma_{3}$. The intermediate principal stress is tentatively defined as:

$$
\sigma_{2}=v\left(\sigma_{1}+\sigma_{3}\right)
$$

Substituting eq. (21) into eqs. (9) and (10), then eqs. (22) and (23) can be obtained:

If $\sigma_{2} \leq \frac{\sigma_{1}+\sigma_{3}}{2}-\frac{\sigma_{1}-\sigma_{3}}{2} \sin \phi$, 
Then,

$$
\begin{aligned}
\sigma_{1}[2(1-\sin \phi)(1+b)-2 v b(1+\sin \phi)] \\
-(1+\sin \phi)(2+2 v b) \sigma_{3}=4 c(1+b) \cos \phi
\end{aligned}
$$

Else,

$$
\begin{aligned}
& (2+2 b v)(1-\sin \phi) \sigma_{1}+ \\
& \quad[2 v b(1-\sin \phi)-2(1+\sin \phi)(1+b)] \sigma_{3}=4 c(1+b) \cos \phi
\end{aligned}
$$

Based on the UST and plane strain assumption, we derive the passive earth pressure in two different cases:

\section{FORMULA DERIVATION OF $P_{P}$ IF}

$$
\sigma_{2} \leq \frac{\sigma_{1}+\sigma_{3}}{2}-\frac{\sigma_{1}-\sigma_{3}}{2} \sin \phi
$$

\section{Formula Derivation of $p_{p}$}

If $\sigma_{2} \leq \frac{\sigma_{1}+\sigma_{3}}{2}-\frac{\sigma_{1}-\sigma_{3}}{2} \sin \phi$,

Minimum principal stress $\sigma_{3}$ can be obtained according to eq. (22):

$\sigma_{3}=\frac{\sigma_{1}(1+b-\sin \phi-b \sin \phi-b v-b v \sin \phi)-2 c(1+b) \cos \phi}{(1+\sin \phi)(1+v b)}$

Uniting eqs. (18) and (25), the following equation is obtained:

$$
\begin{aligned}
\sigma_{1}^{2}- & \sigma_{1} \frac{2 c(1+b) \cos \phi+\gamma z \cos ^{2} \beta(2+b-b \sin \phi)}{1+b-\sin \phi-b \sin \phi-b v-b v \sin \phi}+ \\
& \frac{2 \gamma z \cos ^{2} \beta c(1+b) \cos \phi+\gamma^{2} z^{2} \cos ^{2} \beta(1+\sin \phi)(1+v b)}{1+b-\sin \phi-b \sin \phi-b v-b v \sin \phi}=0
\end{aligned}
$$

Eq. (26) is a unary quadratic equation of $\sigma_{1}$, we here introduce parameters of $G$ and $Q$,

$$
\begin{aligned}
& G=\frac{2 c(1+b) \cos \phi+\gamma z \cos ^{2} \beta(2+b-b \sin \phi)}{1+b-\sin \phi-b \sin \phi-b v-b v \sin \phi} \\
& Q=\frac{2 \gamma z \cos ^{2} \beta c(1+b) \cos \phi+\gamma^{2} z^{2} \cos ^{2} \beta(1+\sin \phi)(1+v b)}{1+b-\sin \phi-b \sin \phi-b v-b v \sin \phi}
\end{aligned}
$$

So eq. (25) can also be expressed as:

$$
\sigma_{1}^{2}-G \cdot \sigma_{1}+Q=0
$$

And its solution is

$$
\sigma_{1}=\frac{\mathrm{G} \pm \sqrt{G^{2}-4 Q}}{2}
$$

Here, we have to make sure that discriminant of the square root is positive $\left(G^{2}-4 Q>0\right)$ in order to get the real root. Obviously, the inclined angle should be bigger than the internal friction angle.
Substituting eq. (28) into eq. (24), we can obtain:

$$
\begin{array}{r}
\sigma_{3}=\frac{\left(G \pm \sqrt{G^{2}-4 Q}\right)(1+b-\sin \phi-b \sin \phi-b v-b v \sin \phi)}{2(1+\sin \phi)(1+v b)} \\
-\frac{2 c(1+b) \cos \phi}{(1+\sin \phi)(1+v b)}
\end{array}
$$

In eq. (28), the positive root $\sigma_{1}$ becomes the minimum principal stress in passive earth pressure circle (while the negative root $\sigma_{1}$ becomes the maximum principal stress in active earth pressure circle); Likewise, in the eq. (29), the bigger $\sigma_{3}$ is the minimum principal stress in passive earth pressure circle, and the other is the maximum principal stress in active earth pressure circle.

In the passive earth pressure circle:

$$
\sigma_{1}+\sigma_{3}=\frac{\left(G+\sqrt{G^{2}-4 Q}\right)(2+b-b \sin \phi)}{2(1+\sin \phi)(1+v b)}-\frac{2 c(1+b) \cos \phi}{(1+\sin \phi)(1+v b)}
$$

Substituting eq. (30) into eq. (20), we can obtain:

$$
p_{\mathrm{p}}=\cos \beta\left[\begin{array}{rr}
\frac{G+\sqrt{G^{2}-4 Q}}{2} & \frac{(2+b-b \sin \phi)}{(1+\sin \phi)(1+v b)} \\
-\frac{2 c(1+b) \cos \phi}{(1+\sin \phi)(1+v b)}
\end{array}\right]-\gamma z \cos \beta
$$

Eq. (31) can be used to calculate the passive earth pressure strength with an inclined cohesive backfill considering the effect of the intermediate principal stress.

Cases of $p_{p}$ when,

$$
\sigma_{2} \leq \frac{\sigma_{1}+\sigma_{3}}{2}-\frac{\sigma_{1}-\sigma_{3}}{2} \sin \phi
$$

The validity of eq. (31) can be verified for simplified soil-structure scenarios.

\section{Case A}

Cohesive soil with horizontal backfill surface behind the wall not considering the intermediate principal stress

Substituting $\beta=0, b=0$ in eqs. (26) and (27), we get

$$
\begin{aligned}
G & =\frac{2 c(1+b) \cos \phi+\gamma z \cos ^{2} \beta(2+b-b \sin \phi)}{1+b-\sin \phi-b \sin \phi-b v-b v \sin \phi}=\frac{2 c \cos \phi+2 \gamma z}{1-\sin \phi} \\
Q & =\frac{2 \gamma z \cos ^{2} \beta c(1+b) \cos \phi+\gamma^{2} z^{2} \cos ^{2} \beta(1+\sin \phi)(1+v b)}{1+b-\sin \phi-b \sin \phi-b v-b v \sin \phi} \\
& =\frac{2 \gamma z c \cos \phi+\gamma^{2} z^{2}(1+\sin \phi)}{1-\sin \phi}
\end{aligned}
$$

Then,

$\frac{G+\sqrt{G^{2}-4 Q}}{2}=\frac{2 c \cos \phi+\gamma z(1+\sin \phi)}{1-\sin \phi}$ 
And

$$
\sigma_{1}+\sigma_{3}=\frac{\left(G+\sqrt{G^{2}-4 Q}\right)(2+b-b \sin \phi)}{2(1+\sin \phi)(1+v b)}-\frac{2 c(1+b) \cos \phi}{(1+\sin \phi)(1+v b)}
$$

Substitute eq. (35) into eq. (31) when $\beta=0, b=0$ :

$$
\begin{aligned}
p_{p} & =\frac{G+\sqrt{G^{2}-4 Q}}{2} \frac{(2+b-b \sin \phi) \cos \beta}{(1+\sin \phi)(1+v b)}-\frac{2 c \cos \phi}{(1+\sin \phi)}-\gamma z \\
& =\gamma z \tan ^{2}\left(45^{\circ}+\frac{\phi}{2}\right)+2 c \tan \left(45^{\circ}+\frac{\phi}{2}\right)
\end{aligned}
$$

Which is identical to Bell's expressions for cohesive soils, eqs. (3) and (4).

\section{Case B}

Granular soil with an inclined backfill surface not considering the intermediate principal stress (when $b=0$ )

Substituting $\mathrm{c}=0, b=0$ in eqs. (26) and (27), we get

$$
\begin{aligned}
G & =\frac{2 c(1+b) \cos \phi+\gamma z \cos ^{2} \beta(2+b-b \sin \phi)}{1+b-\sin \phi-b \sin \phi-b v-b v \sin \phi}=\frac{2 \gamma z \cos ^{2} \beta}{1-\sin \phi} \\
Q & =\frac{2 \gamma z \cos ^{2} \beta c(1+b) \cos \phi+\gamma^{2} z^{2} \cos ^{2} \beta(1+\sin \phi)(1+v b)}{1+b-\sin \phi-b \sin \phi-b v-b v \sin \phi} \\
& =\frac{\gamma^{2} z^{2} \cos ^{2} \beta(1+\sin \phi)}{1-\sin \phi}
\end{aligned}
$$

And

$\frac{G+\sqrt{G^{2}-4 Q}}{2}=\gamma z \cos \beta \frac{\cos \beta+\sqrt{\cos ^{2} \beta-\cos ^{2} \phi}}{1-\sin \phi}$

Substitute eq. (39) into eq. (31) when $\mathrm{c}=0, b=0$ :

$$
\begin{aligned}
& p_{\mathrm{p}}=\frac{G+\sqrt{G^{2}-4 Q}}{2} \frac{(2+b-b \sin \phi) \cos \beta}{(1+\sin \phi)(1+v b)}-\frac{2 c \cos \phi \cos \beta}{(1+\sin \phi)}-\gamma z \cos \beta \\
& =\gamma z \cos \beta \frac{\cos \beta+\sqrt{\cos ^{2} \beta-\cos ^{2} \phi}}{\cos \beta-\sqrt{\cos ^{2} \beta-\cos ^{2} \phi}} \\
& =\sigma_{z} \frac{\cos \beta+\sqrt{\cos ^{2} \beta-\cos ^{2} \phi}}{\cos \beta-\sqrt{\cos ^{2} \beta-\cos ^{2} \phi}}
\end{aligned}
$$

Which is identical to Rankine's expressions with an inclined backfill surface for cohesionless soils, eqs. (1) and (2). Obviously, we have to make sure that discriminant of the square root is positive $\left(\cos ^{2} \beta-\cos ^{2} \phi>0\right)$ in order to get the real root, the inclined angle should be smaller than the internal friction angle from eq. (40).

Formula Derivation of $p_{p}$ if

$$
\sigma_{2} \geq \frac{\sigma_{1}+\sigma_{3}}{2}-\frac{\sigma_{1}-\sigma_{3}}{2} \sin \phi
$$

\section{Formula Derivation of Passive Earth Pressure $p_{p}$}

The derivation process of the section, very similar to section 4 , is as follows:
According to eq. (23) maximum principal stress $\sigma_{1}$ can be obtained,

$$
\sigma_{1}=\frac{\sigma_{3}(1+b+\sin \phi+b \sin \phi-b v+b v \sin \phi)+2 c(1+b) \cos \phi}{(1-\sin \phi)(1+v b)}
$$

Based on eqs. (18) and (41), the following expression is obtained:

$$
\begin{aligned}
\sigma_{3}^{2}- & \sigma_{3} \frac{\gamma z \cos ^{2} \beta(2+b+b \sin \phi)-2 c(1+b) \cos \phi}{1+b+\sin \phi+b \sin \phi-b v+b v \sin \phi} \\
+ & \frac{\gamma^{2} z^{2} \cos ^{2} \beta(1-\sin \phi)(1+v b)-2 \gamma z c(1+b) \cos \phi \cos ^{2} \beta}{1+b+\sin \phi+b \sin \phi-b v+b v \sin \phi}=0
\end{aligned}
$$

Eq. (42) is a unary quadratic equation about $\sigma_{3}$, we introduce another two parameters $G^{\prime}$ and $Q^{\prime}$ :

$$
\begin{aligned}
G^{\prime}= & \frac{\gamma z \cos ^{2} \beta(2+b+b \sin \phi)-2 c(1+b) \cos \phi}{1+b+\sin \phi+b \sin \phi-b v+b v \sin \phi} \\
Q^{\prime}= & \frac{\gamma^{2} z^{2} \cos ^{2} \beta(1-\sin \phi)(1+v b)}{1+b+\sin \phi+b \sin \phi-b v+b v \sin \phi} \\
& -\frac{2 \gamma z c(1+b) \cos \phi \cos ^{2} \beta}{1+b+\sin \phi+b \sin \phi-b v+b v \sin \phi}
\end{aligned}
$$

So the solution to eq. (42) about $\sigma_{3}$ is obtained:

$$
\sigma_{3}=\frac{\left(G^{\prime} \pm \sqrt{G^{\prime 2}-4 Q^{\prime}}\right)}{2}
$$

Here, we, also, have to make sure that discriminant of the square root is positive $\left(G^{2}-4 Q>0\right)$ in order to get the real root. Obviously, the inclined angle should be smaller than the internal friction angle.

From eqs. (42) and (45),

$$
\begin{array}{r}
\sigma_{1}=\frac{\left(G^{\prime} \pm \sqrt{G^{\prime 2}-4 Q^{\prime}}\right)(1+b+\sin \phi+b \sin \phi-b v+b v \sin \phi)}{2(1-\sin \phi)(1+v b)} \\
+\frac{2 c(1+b) \cos \phi}{(1-\sin \phi)(1+v b)}
\end{array}
$$

Similarly, in eq. (45), the positive root $\sigma_{3}$ becomes the minimum principal stress in passive earth pressure circle, (while the negative root $\sigma_{3}$ becomes the minimum principal stress in active earth pressure circle); Likewise, in the eq. (46), the bigger $\sigma_{1}$ is the maximum principal stress in passive earth pressure circle, and the other is the maximum principal stress in active earth pressure circle.

In passive earth pressure state: 


$$
\sigma_{1}+\sigma_{3}=\frac{\frac{G^{\prime}+\sqrt{G^{\prime 2}-4 Q^{\prime}}}{2}(2+b+b \sin \phi)+2 c(1+b) \cos \phi}{(1-\sin \phi)(1+v b)}
$$

Finally, the passive earth pressure is obtained:

$$
\begin{aligned}
& p_{p}=\frac{\frac{G^{\prime}+\sqrt{G^{\prime 2}-4 Q^{\prime}}}{2}(2+b+b \sin \phi)+2 c(1+b) \cos \phi}{(1-\sin \phi)(1+v b)} \cos \beta \\
& -\gamma z \cos \beta
\end{aligned}
$$

Cases of $\mathbf{p}_{\mathbf{p}}$ when $\sigma_{2} \geq \frac{\sigma_{1}+\sigma_{3}}{2}-\frac{\sigma_{1}-\sigma_{3}}{2} \sin \phi$

In order to verify the validity of eq. (48), there are simplified soil-structure scenarios.

\section{Case A}

Cohesive soil with horizontal backfill surface behind the wall not considering the intermediate principal stress

Substituting $\beta=0, b=0$ in eqs. (44) and (45), we get

$$
\begin{aligned}
G^{\prime} & =\frac{\gamma z \cos ^{2} \beta(2+b+b \sin \phi)-2 c(1+b) \cos \phi}{1+b+\sin \phi+b \sin \phi-b v+b v \sin \phi} \\
& =\frac{2 \gamma z-2 c \cos \phi}{1+\sin \phi} \\
Q^{\prime} & =\frac{\gamma^{2} z^{2} \cos ^{2} \beta(1-\sin \phi)(1+v b)}{1+b+\sin \phi+b \sin \phi-b v+b v \sin \phi} \\
& -\frac{2 \gamma z c(1+b) \cos \phi \cos ^{2} \beta}{1+b+\sin \phi+b \sin \phi-b v+b v \sin \phi} \\
= & \frac{\gamma^{2} z^{2}(1-\sin \phi)-2 \gamma z c \cos \phi}{1+\sin \phi}
\end{aligned}
$$

Then,

$$
\frac{G^{\prime}+\sqrt{G^{2}-4 Q^{\prime}}}{2}=\gamma z
$$

Substituting eq. (51) into eq. (48) when $\beta=0, b=0$, we obtain:

$$
\begin{aligned}
p_{\mathrm{p}} & =\frac{G^{\prime}+\sqrt{G^{\prime 2}-4 Q^{\prime}}}{2} \cdot \frac{(2+b-b \sin \phi)}{(1-\sin \phi)(1+v b)}+\frac{2 c(1+b) \cos \phi}{(1-\sin \phi)(1+v b)}-\gamma z \\
& =\gamma z \tan ^{2}\left(45^{\circ}+\frac{\phi}{2}\right)+2 c \tan \left(45^{\circ}+\frac{\phi}{2}\right)
\end{aligned}
$$

Which is identical to Bell's expressions for cohesive soils with a horizontal backfill surface, eqs. (3) and (4).

\section{Case B}

Cohesionless soil with an inclined backfill surface not considering the intermediate principal stress (when $b=0$ )

Substituting $\mathrm{c}=0, b=0$ in eqs. (44) and (45), we get,

$$
G^{\prime}=\frac{2 c(1+b) \cos \phi+\gamma z \cos ^{2} \beta(2+b-b \sin \phi)}{1+b-\sin \phi-b \sin \phi-b v-b v \sin \phi}=\frac{2 c \cos \phi+2 \gamma z \cos ^{2} \beta}{1-\sin \phi}
$$

$$
\begin{aligned}
Q^{\prime} & =\frac{2 \gamma z \cos ^{2} \beta c(1+b) \cos \phi+\gamma^{2} z^{2} \cos ^{2} \beta(1+\sin \phi)(1+v b)}{1+b-\sin \phi-b \sin \phi-b v-b v \sin \phi} \\
& =\frac{\gamma^{2} z^{2}(1+\sin \phi)}{1-\sin \phi}
\end{aligned}
$$

Then,

$$
\frac{G^{\prime}+\sqrt{G^{12}-4 Q^{\prime}}}{2}=\frac{\gamma z \cos \beta\left(\cos \beta+\sqrt{\cos ^{2} \beta-\cos ^{2} \phi}\right)}{1+\sin \phi}
$$

Substituting eq. (55) into eq. (48), we obtain:

$$
\begin{aligned}
p_{\mathrm{p}} & =\left(\frac{G^{\prime}+\sqrt{G^{\prime 2}-4 Q^{\prime}}}{2} \cdot \frac{(2+b-b \sin \phi)}{(1-\sin \phi)(1+v b)}+\frac{2 c(1+b) \cos \phi}{(1-\sin \phi)(1+v b)}\right) \cos \beta \\
& =\gamma z \cos \beta \frac{\cos \beta+\sqrt{\cos ^{2} \beta-\cos ^{2} \phi}}{\cos \beta-\sqrt{\cos ^{2} \beta-\cos ^{2} \phi}}
\end{aligned}
$$

Which agrees with Rankine's expressions for cohesionless soils with an inclined backfill surface, eqs. (1) and (2). Obviously, we have to make sure that discriminant of the square root is positive $\left(\cos ^{2} \beta-\cos ^{2} \phi>0\right)$ in order to get the real root, the inclined angle should be smaller than the internal friction angle from eq. (56).

\section{COMPARISON BETWEEN THE NEW RESULTS AND THE CLASSICAL ONES}

In order to show the differences between the classical $(b=0)$ and the present magnitudes of the earth pressure, we, firstly, should know whether $\sigma_{2}$ is bigger than $\frac{\sigma_{1}+\sigma_{3}}{2}+\frac{\sigma_{1}-\sigma_{3}}{2} \sin \phi$ or not. The result counts on the values of Poisson's Ratio $(v)$ and internal friction angle $(\phi)$. The value of $v$ is, mostly, less than 0.5 , so $\sigma_{2}$ is the smaller one. Two cases are listed as follow:

\section{Case A: Comparison between the New Results and Rankin's Theory}

There are three figures (Figs.4, $\mathbf{5}$ and $\mathbf{6}$ ) to compare the new results with Rankin's theory by changing different parameters. The results show that passive earth pressures computed by Rankin's theory are obviously higher than the new results. The pressures become smaller when $b$ increases from Fig. (4). The pressures change slightly with Poisson's ratio increasing from Fig. (5).

\section{Case B: Comparison between the New Results and Bell's Equation}

Similarly, we make another three figures (Figs. 7, 8, and 9) to compare the new results with Bell's equation by changing different parameters. The results show that passive earth pressures computed by Bell's theory are obviously higher than the new results. From the figures, the pressures become smaller when $b$ increases. The pressures decrease 


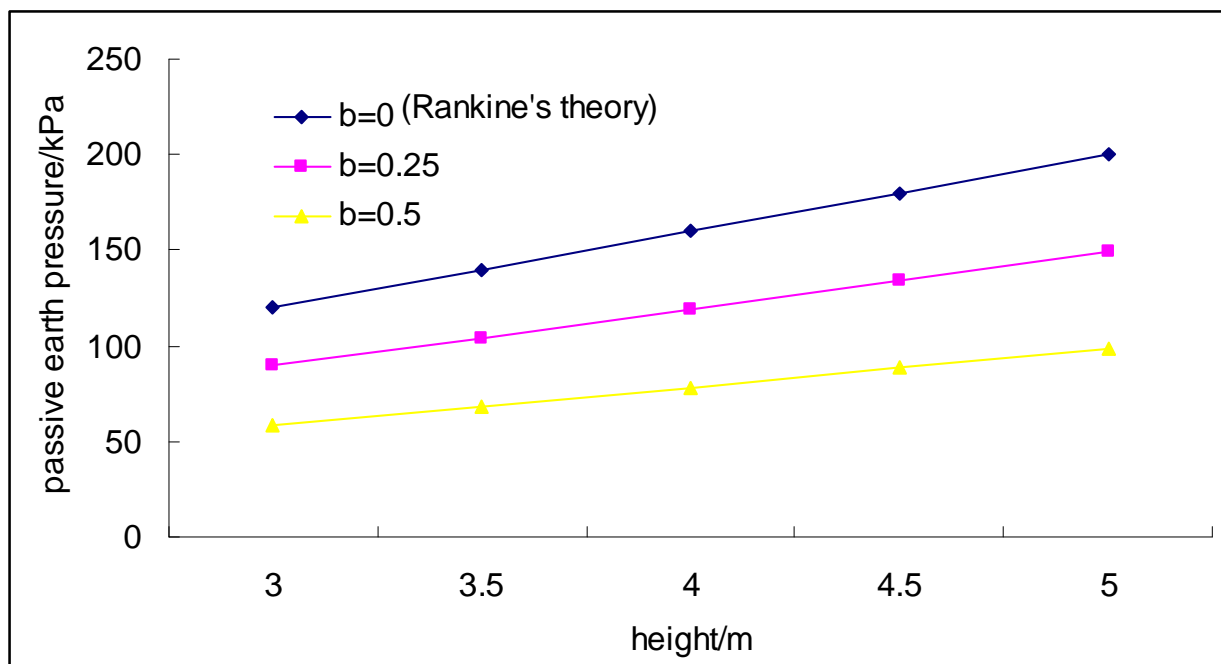

Fig. (4). Pressures at different heights changing with $b$.

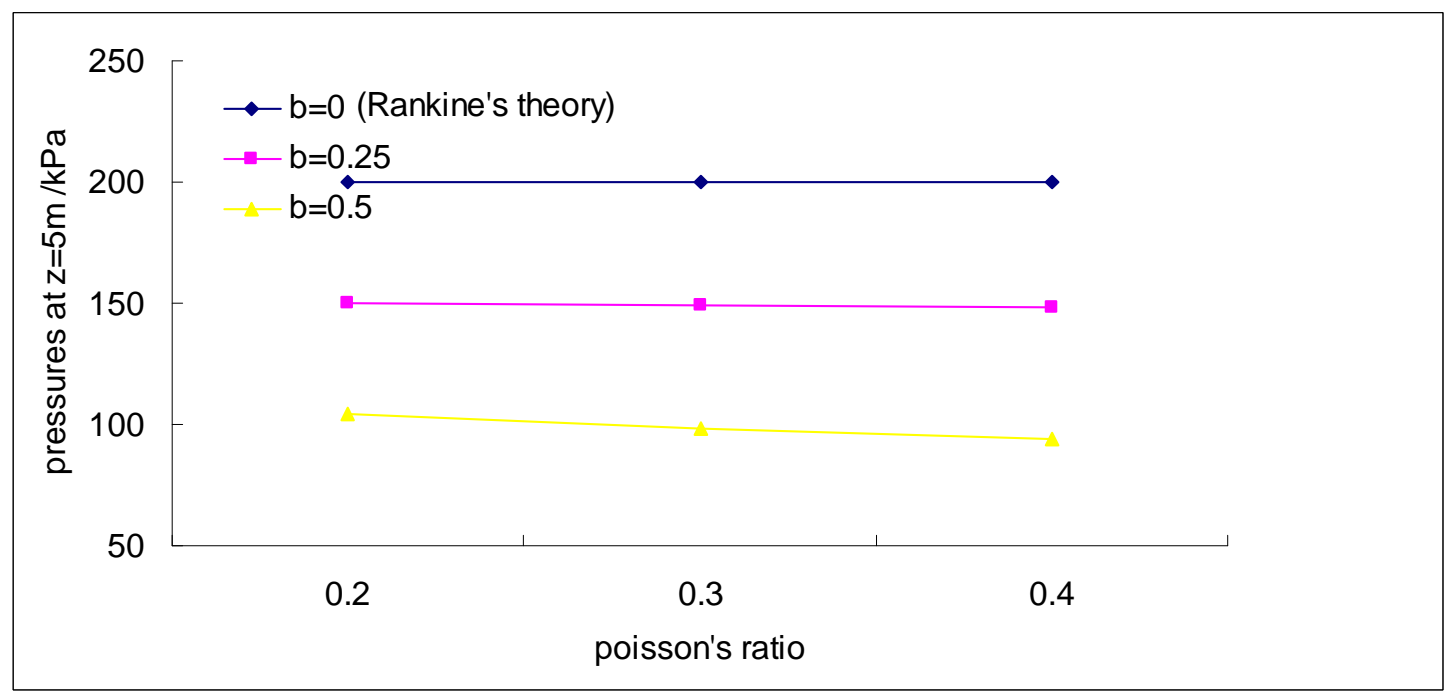

Fig. (5). Pressures of different Poisson's Ratios changing with b.

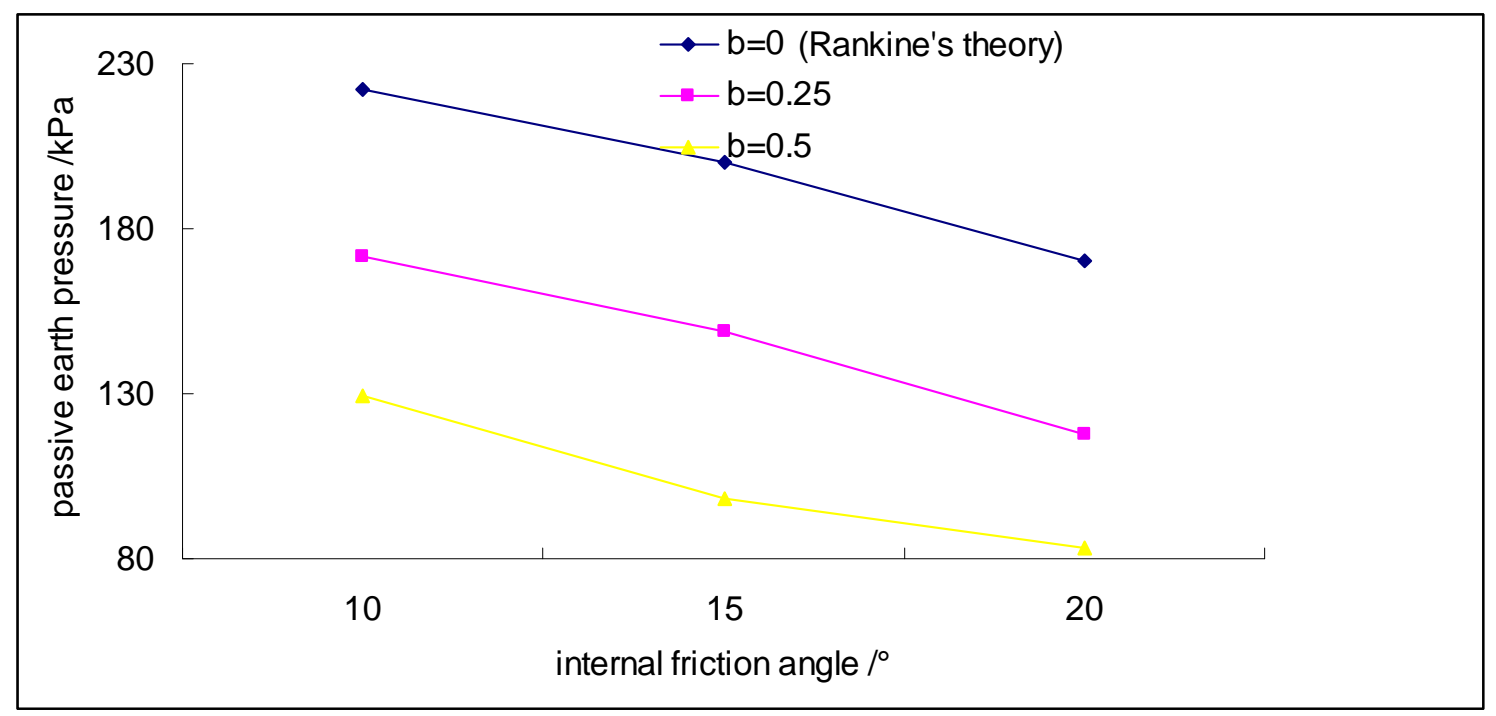

Fig. (6). Pressures of different internal friction angles changing with $b$. 
slightly with Poisson's ratio changing from Fig. (8), and greatly with internal friction angle changing from Fig. (9).

\section{CONCLUSIONS AND DISCUSSIONS}

An analytical solution has been developed to determine the passive lateral earth pressure distribution on a retaining



Fig. (7). Pressures at different heights changing with $b$.

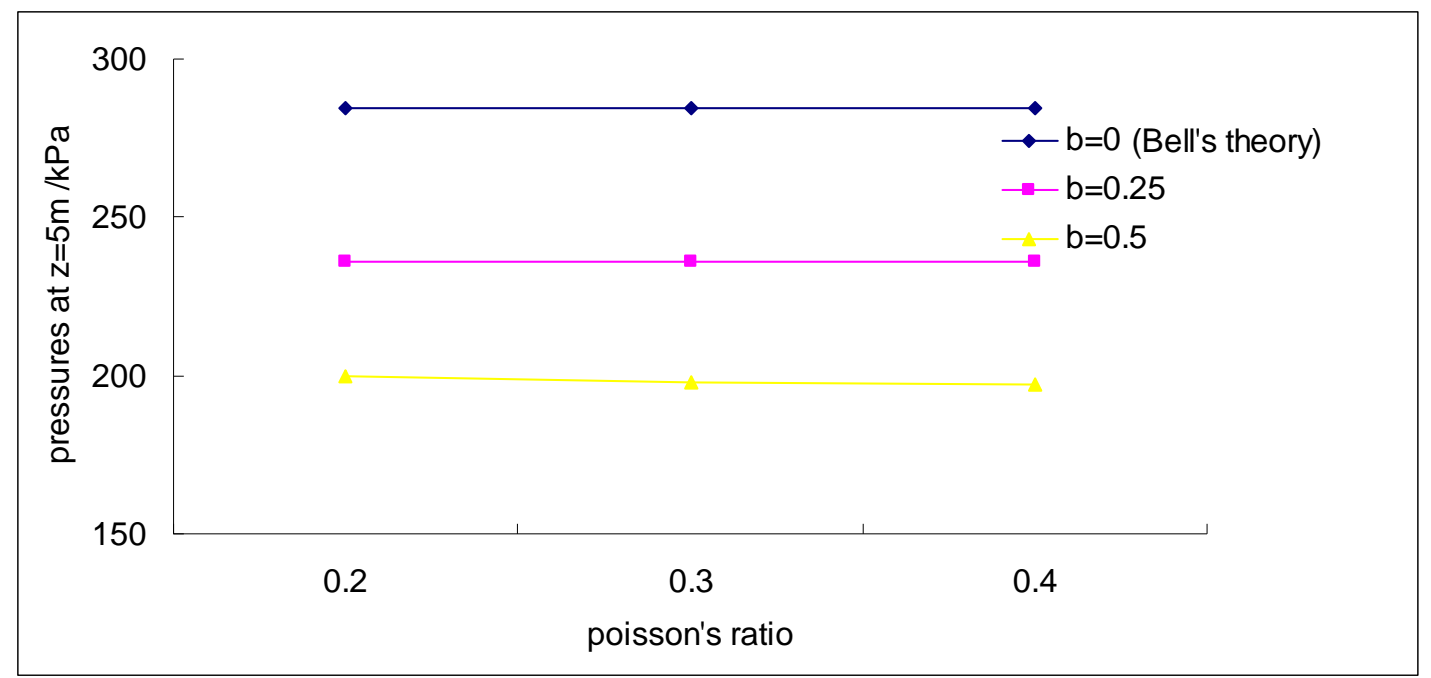

Fig. (8). Pressures of different Poisson's Ratios changing with b.

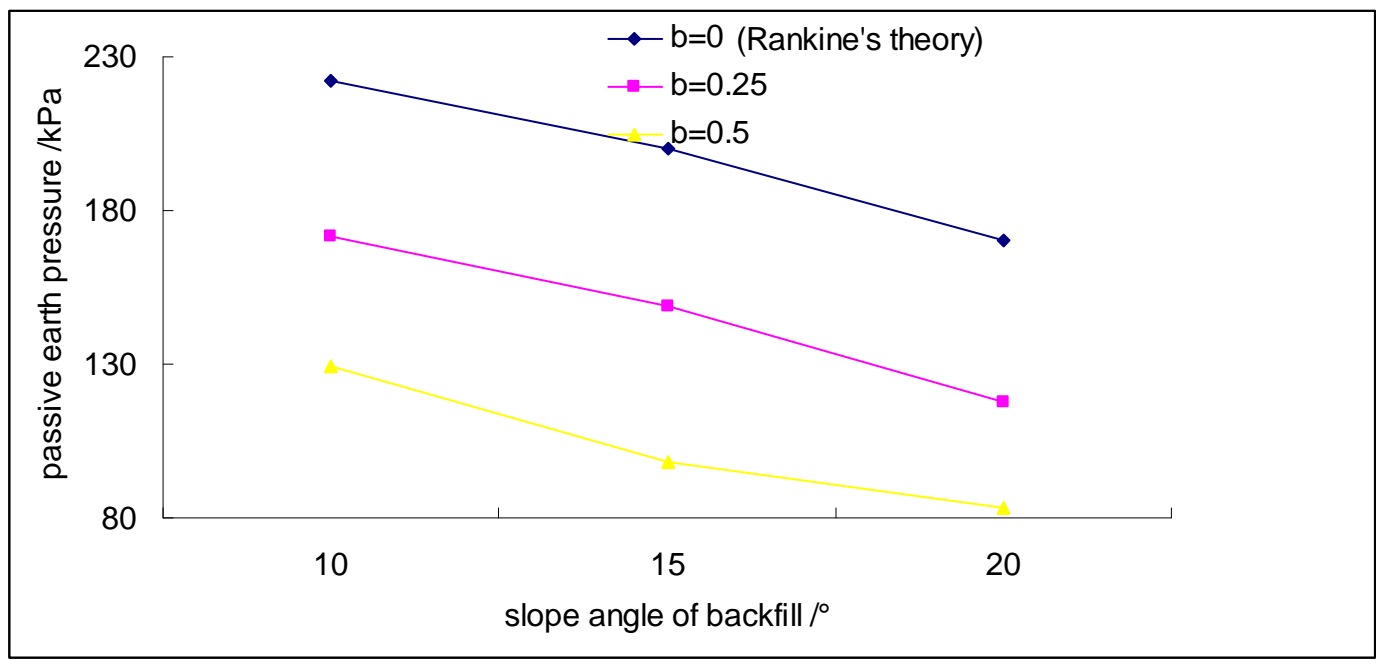

Fig. (9). Pressures of different slope angles changing with $b$. 
wall with a cohesive inclined backfill considering the effect of the intermediate principal stress based on UST and the plane strain assumption. The results show that the Poisson's Ratio, $v$, has an slight effect on the earth pressure while coefficient of intermediate principal stress, $b$, has an big effect on the earth pressure. The analytical solution is a generalized expression that can be used to for retaining structures against inclined various soil and rock backfills. The results show that the analytical solution encompasses both Rankine's solution and Bell's equation.

However, it should be noted that the principal stress $\sigma_{\mathrm{y}}$ be not always the intermediate principal in the direction of $\varepsilon_{\mathrm{y}}=0$. Only in the plane strain condition, the principal stress $\sigma_{y}$ is always the intermediate principal in the direction of plane strain. It is a pity that, in this paper, there is no comparison between the calculated earth pressure and the actual earth pressure, mostly because the actual earth pressure is hard to be measured.

\section{ACKNOWLEDGEMENTS}

The authors acknowledge financial support from Chinese National Science and Technology Support Program (No. 2008BAK50B04-3), key project (No. KZCX2-YW-Q03-2), ([2009]03-02-03) and National Natural Science Foundation of China (No. 41072226).

\section{LIST OF SYMBOLS}

$$
\begin{aligned}
& \sigma_{1}=\text { Maximum principal stress } \\
& \sigma_{2}=\text { Intermediate principal stress } \\
& \sigma_{3}=\text { Minimum principal stress } \\
& b=\text { Coefficient of intermediate principal shear stress } \\
& \sigma_{t}=\text { Tensile strength } \\
& \sigma_{c}=\text { Compressive strength } \\
& c=\text { Soil cohesion } \\
& \phi=\text { Internal friction angle of soil } \\
& \lambda=\text { The ratio of tensile strength } \sigma_{t} \text { and compressive } \\
& \alpha=\text { strength } \sigma_{\mathrm{c}} \\
& \varepsilon_{y}=\text { Angle that the failure surface makes with the } \\
& \text { horizontal }
\end{aligned}
$$

$$
\begin{aligned}
& \mathrm{E}=\text { Modulus of elasticity } \\
& \gamma=\text { Unit weight of soil } \\
& v=\text { Poisson's Ratio of filling, } 0<v<0.5 \\
& \beta=\text { Angle that backfill makes with the horizontal } \\
& P_{\mathrm{p}}=\text { Passive lateral earth pressure } \\
& \tau=\text { Shear strength of soil } \\
& \sigma=\text { Lateral stress in soil } \\
& \sigma_{\mathrm{z}}=\text { Vertical stress in soil at depth of } \\
& z=\text { Depth below ground surface }
\end{aligned}
$$

\section{REFERENCES}

[1] K. Terzaghi, R. B. Pceck and G. Mesri, Soil Mechanics in Engineering Practice. New York: John Wiley and Sons, Inc., 1996.

[2] A. Caquot, and J. Kerisel, Tables for the Calculation of the Passive Pressure, Active Pressure and Bearing Capacity of Foundations. Paris: Gauthier-Villars, 1948.

[3] V. V. Sokolovski, Statics of Soil Media, 2nd ed. Butterworth \& Co. Ltd: London, 1960.

[4] K. Habibagahi and A. Ghahramani, "Zero extension line theory of earth pressure", Journal of the Geotechnical Engineering Division, $A S C E$, vol. 105, no. GT7, pp. 881-896, 1979.

[5] NAVFAC. DM7.2: Foundations and Earth Structures. Naval Facilities Engineering Command, United States Department of the Navy, Alexandria, Va., 1982.

[6] M. H. Yu, New System of Strength Theory. Xi'an: Xi'an Jiaotong University Press: 1992 (in Chinese).

[7] M. H. Yu, "Unified strength theory for geomaterials and its application", Chinese Journal of Geotechnical Engineering, vol. 16, no. 2, pp. 1-9, 1994.

[8] M. H. Yu, "Twin shear stress yield criterion", International Journal of Mechanical Sciences, vol. 25, no. 1, pp. 71-74, 1983.

[9] M. H. Yu, L. N. He and L. Y. Song, "Twin shear stress theory and its generalization", Scientia Sinica (Series A), vol. 28, no. 12, pp. 1174-1183, 1985 (in Chinese).

[10] G. W. Ma, and I. Shouju, "Plastic limit analysis of circular plates with respect to unified yield criterion", International Journal of Mechanical Sciences, vol. 10, no. 10, pp. 963-976, 1998.

[11] G. W. Ma, and H. Hao, "Unified plastic limit analyses of circular plates under arbitrary load", Journal of Applied Mechanics, Vol. 66, no. 2, pp. 568-570, 1999.

[12] M. H. Yu, J. C. Li, and Y. Q. Zhang, "Unified characteristics line theory of spacial axisymmetric plastic problem", Sciences in China (Series E), vol. 44, no. 2, pp. 207-215, 200 (in Chinese).

[13] J. Zhang, X. Wei and R. L. Hu, "Rankine Earth Pressure with an Inclined Backfill Surface Considering the Effect of the Intermediate Principal Stress", in extended abstracts to the proceedings of the $11^{\text {th }}$ congress of the IAEG geologically active, 2010, pp. 3945 3952. 